\title{
Millimetric observations of the SZE towards Corona Borealis Supercluster
}

\author{
Gemma Luzzi* \\ Department of Physics, University "La Sapienza", P.le A. Moro 2, 00185, Rome, Italy \\ E-mail: Gemma.Luzzi@roma1.infn.it
}

\author{
E.S. Battistelli ${ }^{1,2}$, M. De Petris ${ }^{2}$, L. Lamagna ${ }^{2}$, R.A. Watson ${ }^{3,7}$, R. Rebolo ${ }^{1,4}$, \\ F. Melchiorri ${ }^{2}$, R. Génova-Santos ${ }^{1}$, S. De Gregori², J.A. Rubiño-Martin ${ }^{1}$, \\ R.D. Davies ${ }^{3}$, R.J. Davis ${ }^{3}$, K. Grainge ${ }^{5}$, M.P. Hobson ${ }^{5}$, R.D.E. Saunders ${ }^{5}$, and \\ P.F. Scott ${ }^{5}$ \\ ${ }^{1}$ Instituto de Astrofísica de Canarias, C/Via Lactea s/n, 38200, La Laguna, Spain \\ ${ }^{2}$ Department of Physics, University "La Sapienza", P.le A. Moro 2, 00185, Rome, Italy \\ ${ }^{3}$ Jodrell Bank Observatory, University of Manchester, Macclesfield, Cheshire, SK11 9DL, UK \\ ${ }^{4}$ Consejo Superior de Investigaciones Científicas, Spain \\ ${ }^{5}$ Astrophysics Group, Cavendish Laboratory, University of Cambridge, Cambridge, CB3 OHE, \\ $U K$
}

\begin{abstract}
We have observed the Corona Borealis Supercluster with the Millimeter and Infrared Testa grigia Observatory (MITO) at 143, 214, 272, and $353 \mathrm{GHz}$. We present a description of the measurements, data analysis, and results together with a comparison of observations performed at $33 \mathrm{GHz}$ with the Very Small Array (VSA). Observations have been made in a region of the supercluster corresponding to one Cosmic Microwave Background (CMB) cold spot previously detected in VSA temperature map. Observational strategy and data analysis are described, explaining the procedures used to disentangle primary and secondary anisotropies in the resulting maps. With a data analysis using map making and the maximum entropy method we claim a weak detection of a faint signal spectrally compatible with a SZ effect characterized at most by a Comptonization parameter $y=\left(7.8_{-4.4}^{+5.3}\right) \times 10^{-6} 68 \% \mathrm{CL}$. The low level of confidence in the presence of a SZ signal invites us to study this sky region with higher sensitivity and angular resolution experiments like the already planned upgraded versions of VSA and MITO.
\end{abstract}

$C M B$ and Physics of the Early Universe

20-22 April 2006

Ischia, Italy

*Speaker. 


\section{Introduction}

The Sunyaev-Zel'dovich effect (SZE) [6], arises from the Compton scattering of the CMB radiation by electrons in the hot gas in clusters of galaxies. So far, the SZE has only been observed in the direction of clusters of galaxies; however, other objects may also be sources of detectable SZE. In superclusters of galaxies (SCG) the enhancements of baryon density over the mean cosmological baryon density are expected to be small, but the path lenghts may be so long that a significant SZE builts up. The Corona Borealis Supercluster has been mapped by VSA (Genova Santos et al. 2005). A major result appearing in these maps is the presence of a strong temperature decrement near the center of the SCG in a direction with no known cluster of galaxies. The evidence of this SCG spot being due to inverse Compton scattering would result from a positive signal at frequencies higher than $\sim 217 \mathrm{GHz}$, an effect that uniquely characterizes the SZE. We describe here the observations that have been performed at MITO in 4 high frequency bands with the aim of disentangling sources of anisotropy with different spectral behavior .

\section{MITO observations}

\subsection{The instrument}

MITO (Millimetre and Infrared Testagrigia Observatory) [2] telescope has a Cassegrain configuration, i.e. two-element system with $2.6-\mathrm{m}$ in diameter parabolic primary mirror and $41 \mathrm{~cm}$ in diameter hyperbolic secondary mirror. The telescope is optimized for differential measurements, by choosing the position of the wobbling axis of the secondary mirror around the neutral point with digitally controlled wave-forms. The pointing system is based on an altazimuthal mount, mainly due to the need of performing sky modulation at constant elevation in order to reduce atmospheric contributions. FotoMITO is a multichannel single pixel photometer with a f.o.v. of 16 arcminutes FWHM. It works in 4 bands centered at 143, 214, 272, and $353 \mathrm{GHz}$. The detectors are 4 standard composite bolometers with sapphire absorbers and NTD Ge thermistors. Detectors are coupled to radiation via multi-mode Winston cones allowing maximum collection efficiency within the field of view. The cold optics is realized by spherical mirrors, Winston cones and band-pass freestanding meshes as dichroics for spectral selection. A double stage ${ }^{3} \mathrm{He}-{ }^{4} \mathrm{He}$ fridge allows a working temperature of $290 \mathrm{mK}$ with a stability of the order of $0.2 \mathrm{mK} / \mathrm{h}$ for more than 80 hours operation [4]. The bands of operation, about 10\%-20\% wide (FWHM), have been chosen to match both the atmospherical transparency windows in the $\mathrm{mm} /$ submm region and the main features of the $\mathrm{S}-\mathrm{Z}$ effect. In particular the second channel has been selected to match the SZE crossover frequency, while the fourth channel serves as "spectral backup" in order to monitor the foreground and the local emission from the atmosphere.

\subsection{Observing strategy}

Data were collected in March 2004 and include observations on the deepest spot of the VSA map (i.e. spot H), calibration scans on Jupiter, some pointings on Saturn and Tau A used as secondary calibration sources, and routine skydips to extract information about atmospheric emission. Sky modulation has been performed by a 3-fields square-wave-like scanning along constant elevation followed by lock-in demodulation. This strategy allows efficient removal of the atmosphere 
emission even when it exhibits strong linear gradients parallel to the horizon. The beamthrow was set to 41 arcminutes and the modulation frequency to $4.5 \mathrm{~Hz}$ with 2 nd harmonic demodulation. Measurements were carried out by performing drift scans (DSs) on the source. This procedure allows to reduce the bolometer's microphonics being the telescope at rest during the observations. We have collected 105 scans across the VSA H spot in the Corona Borealis region. Each scan is 10 minutes long in RA.

\section{Data analysis}

\subsection{Noise filtering and atmospherical decorrelation}

In order to cope with the atmosphere emission fluctuations a decorrelation technique is applied on data. It is possible thanks to the superposition of the four MITO channels field of view (total beam overlapping) and to the presence of the $353 \mathrm{GHz}$ channel which is more sensitive to the atmospherical emission. The basic idea is to subtract from the channels at the frequency of cosmological interest $\left(v_{s}\right)$ the channel most affected by atmospherical emission $\left(v_{a}\right)$ after having multiplied it by the correlation coefficient $a_{a s}$. A key ingredient, necessary in order to perform an efficient decorrelation, is that the various drift scans should be characterized by high correlation between channels. Uncorrelated signals may be due to different optical depths for different spectral channels, to fluctuations at different altitudes, and to differences in the channel beam [5]. An additional filtering procedure, presented in [5], has been applied to those scans showing moderately high correlation even if not enough to undergo an efficient decorrelation. The procedure consists in passing data through a non-linear amplifier that allows us to "clean" our data from non-gaussianity in an unbiased way controlling the systematics.

\subsection{Maximum entropy method}

As map reconstruction is degenerate for differential and interferometric observations, an extra regularizing constraint is required which is conveniently provided by the Maximum Entropy Method (MEM). In order to extract the best representative signal maps from the original data sets, we have carried out the MEM reconstruction for both the MITO decorrelated scans and the gridded VSA visibilities for the observed spot in Corona Borealis supercluster. We used the same method of fitting modeled data resulting from a trial sky for both data sets in order to be sure of consistency. The sky model used is comprised of a 70 by 20 pixel image covering RA $15^{\mathrm{h}} .3 \div 15^{\mathrm{h}} .5$ and Dec $28^{\circ} .5 \div 29^{\circ} .3$, in which each pixel brightness represents a free parameter to be found. An iterative gradient method was used to update the sky model and in turn calculate the model scans and visibilities. A maximum likelihood analysis was then applied pixel by pixel on the extracted maps in order to derive information about the presence of primary and secondary anisotropies in our data.

\section{Results}

The maximum likelihood analysis on the maps extracted by the MEM has been performed in two steps: by first extracting common signals from the different observational channels, and then by identifying the frequency dependent signals. As a result, we find evidence of a small 

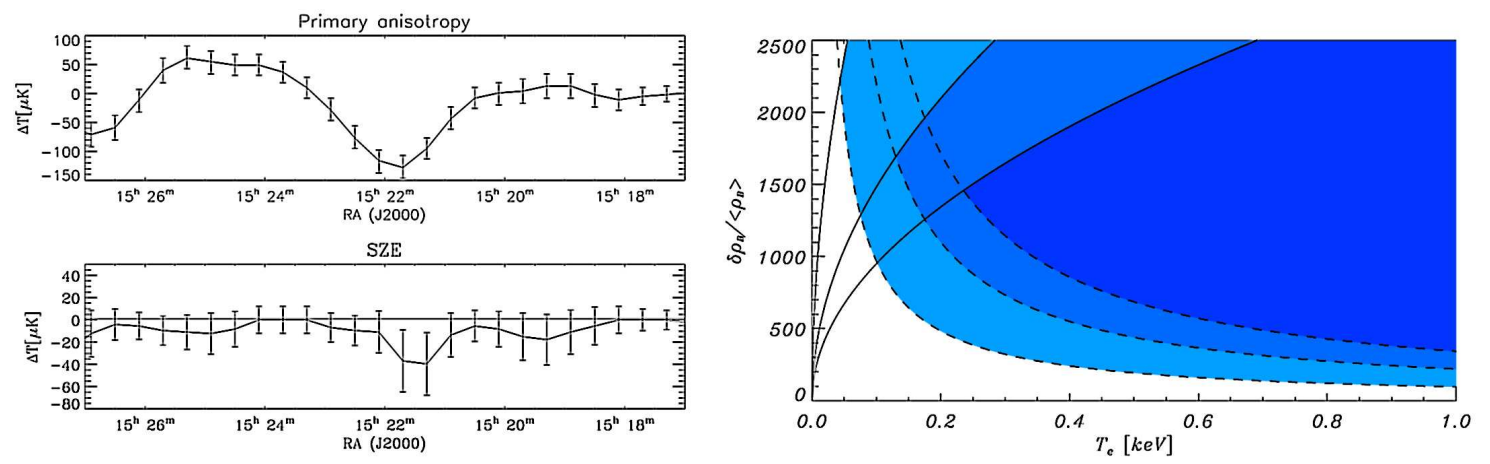

Figure 1: Left: a cut at $\delta=28^{\circ} 00^{\prime} 24^{\prime \prime}$ of the derived anisotropy maps extracted for primary and secondary contribution. A minimum is evident in the lower plot corresponding to a RJ temperature of $\Delta \mathrm{T}_{\mathrm{SZ}}=\left(-42_{-29}^{+24}\right) \mu \mathrm{K}(\mathrm{RJ})$ with a primary anisotropy value of $\Delta \mathrm{T}_{\mathrm{ani}}=\left(-128_{-18}^{+21}\right) \mu \mathrm{K}$ [1]. Right: Constraints placed in the overdensity (with respect to the mean baryon density) and electron temperature space from both the SZ observations (dashed line) and the information extracted from the upper limit to the X-ray emission - ROSAT R6 band observations- (solid line). Lighter and darker colors correspond to $-1 \sigma$ and $+1 \sigma$ respectively [1].

signal common to all the 4 channels with a spectral behavior typical of the SZE. The maximum signal corresponds to the pixel at coordinates $\mathrm{RA}=15^{\mathrm{h}} 21^{\mathrm{m}} 42^{\mathrm{s}}, \delta=28^{\circ} 00^{\prime} 24^{\prime \prime}$, with a maximum intensity of the comptonization parameter of $y=\left(7.8_{-4.4}^{+5.3}\right) \times 10^{-6} 68 \% \mathrm{CL}\left(\Delta \mathrm{T}_{\mathrm{SZ}}\right.$ of the order of $-40 \mu \mathrm{K}$ (RJ) ) (Fig. 1). The signal observed by VSA is dominated by a primary anisotropy with a small contribution from SZE. Our results show that the primordial CMB component of the spot originally detected by VSA has now an amplitude compatible with Gaussianity. About the SZ component the hypothesis of an unknown unresolved cluster can not be discarded. Anyway, we consider also the case of WHIM (see the contribution "SZE at supercluster scales with PLANCK" by J.A. Rubiño-Martin and references therein) being distributed in a filament, aligned along the line of sight of the supercluster with physical dimension of some $40 \mathrm{Mpc}$. Using the amplitude of the measured SZ component, we can set the constraints in the electron density-temperature plane for such a filament (Fig. 1). If we assume for the gas temperature the typical values predicted for the WHIM $(0.5 \div 0.8 \mathrm{keV})$, then we would expect baryon overdensities greater than $400 \div 600$ times the mean baryon density in the local Universe, implying a baryonic mass content $\left(\sim 3 \times 10^{14} \mathrm{M}_{\odot}\right)$ comparable to the total baryonic mass of the cluster members of the supercluster.

\section{References}

[1] Battistelli, E. S., et al., 2006, ApJ in press [astro-ph/0603702]

[2] De Petris, M., et al., 1999, New Astronomy 43297

[3] Genova-Santos, R., et al., 2005, MNRAS 36379

[4] Maiani, T., et al., 1999, Cryogenics 39459

[5] Melchiorri, F., and Olivo-Melchiorri, B. 2005, Procs. of the International School of Physics "Enrico Fermi" 6-16 July 2004, IOS press Melchiorri and Rephaeli eds 211-223

[6] Sunyaev, R.A. and Zel'dovich, Ya.B. 1972, Comm. Astrphys. Space Phys. 4173 